\title{
Implementación de una máquina didáctica clasificadora para el aprendizaje de procesos mecatrónicos
}

\section{(Implementation of a classifier didactical machine for learning mechatronic processes)}

\author{
De La Cruz Alex¹, Donoso Juan ${ }^{1}$, Oscar Gonzales ${ }^{1}$, Ricardo Soto ${ }^{1}$
}

\begin{abstract}
Resumen:
El presente artículo muestra el diseño y construcción de una máquina didáctica clasificadora de objetos mediante visión artificial. El objetivo de la implementación de la máquina es que esta sea utilizada como módulo de aprendizaje de procesos mecatrónicos. En el proyecto, se describen los aspectos teóricos que relacionan conceptos de diseño mecánico, diseño electrónico y manejo de software los cuales constituyen los ejes de una rama en la ciencia y tecnología en auge, la cual es la mecatrónica. El diseño de la máquina se desarrolló a base de los requerimientos del usuario, a través de la metodología del diseño concurrente, para definir y materializar las soluciones de hardware y software adecuadas. Se implementó el software LabVIEW 2015 para la adquisición y análisis de imágenes en alta velocidad, así como para el establecimiento de la comunicación de datos con un controlador lógico programable (siglas PLC en inglés) vía Ethernet mediante una plataforma de comunicaciones abierta que se conoce en la literatura inglesa como Open Platform Communications - OPC. Además, se utilizó la plataforma Arduino MEGA 2560 para el control del movimiento del motor a pasos y los servomotores del módulo.
\end{abstract}

Palabras clave: visión artificial; comunicación OPC; diseño concurrente.

\begin{abstract}
:
The present article shows the design and construction of a classifier didactical machine through artificial vision. The implementation of the machine is to be used as a learning module of mechatronic processes. In the project, it is described the theoretical aspects that relate concepts of mechanical design, electronic design and software management which constitute popular field in science and technology, which is mechatronics. The design of the machine was developed based on the requirements of the user, through the concurrent design methodology to define and materialize the appropriate hardware and software solutions. LabVIEW 2015 was implemented for high-speed image acquisition and analysis, as well as for the establishment of data communication with a programmable logic controller (PLC) via Ethernet and an open communications platform known as Open Platform Communications - OPC. In addition, the Arduino MEGA 2560 platform was used to control the movement of the step motor and the servo motors of the module. Also, is used the Arduino MEGA 2560 to control the movement of the stepper motor and servo motors in the module. Finally, we assessed whether the equipment meets the technical specifications raised by running specific test protocols.
\end{abstract}

Keywords: artificial vision, machine vision; OPC communication; concurrent design.

\footnotetext{
${ }^{1}$ Escuela Politécnica Nacional, Quito - Ecuador ( \{alex.delacruz, juan.donoso, oscar.gonzales, ricardo.soto\}
} @epn.edu.ec ) 


\section{Introducción}

Los actuales requerimientos de integración de las diferentes ramas de conocimiento en la industria incentivan a que los estudiantes de ingeniería mecánica y afines se involucren más con la naturaleza y el entorno de procesos industriales o mecatrónicos (Bolton, 2001). El proceso de enseñanza aprendizaje en las instituciones de educación superior en temas de automatización conlleva al uso de módulos de entrenamiento que emulen procesos industriales. Sin embargo, en mecatrónica es necesario integrar un conjunto de ramas de la ciencia y tecnología como lo son la mecánica, electrónica y sistemas de información (Bolton, 2001).

Las máquinas clasificadoras de objetos determinan un amplio campo de investigación en lo que corresponde a soluciones para llevar a cabo la separación de objetos bajo diferentes parámetros, tales como: el tamaño, color y forma (Alcalde \& Bone, 2013). Para llegar a este fin, el diseño y materialización de la máquina se realiza a través de la metodología diseño concurrente propuesto por Carles Riba (2002).

El presente trabajo se halla constituido de la siguiente manera: en la Sección 2, se presentan los aspectos teóricos relacionados con la concepción del módulo de entrenamiento, con un enfoque en los aspectos requeridos para clasificar 9 clases de objetos por forma y color; además se presenta la metodología para definir el diseño de la máquina a través del criterio QFD (Quality Function Deployment) propuesto por Riba (2002) así como también la metodología para la validación y selección de componentes mecánicos, eléctricos y electrónicos; en la Sección 3, se discuten los resultados de la definición de la máquina en torno al cumplimiento de las especificaciones técnicas planteadas mediante la ejecución de protocolos de pruebas y en la Sección 4, se presentan las conclusiones y recomendaciones generadas durante el desarrollo del trabajo.

\section{Metodología}

El diseño y construcción de la máquina clasificadora tiene finalidades didácticas que permiten complementar de manera práctica los conocimientos teóricos adquiridos durante la formación académica en las materias como: control automático, PLCs, robótica, además de introducir las ventajas que presenta la visión artificial en la automatización de ciertos procesos.

\subsection{Planteamiento y selección de alternativas de diseño}

\subsubsection{Casa de la calidad}

La casa de la calidad es una herramienta del QFD, mediante la cual se determinan los requerimientos técnicos del producto, al establecer relaciones entre las demandas del cliente y los criterios del ingeniero o especificaciones técnicas del producto (Cintas \& Llabres, 1995). Los compromisos técnicos para el desarrollo de la máquina han sido establecidos siguiendo la metodología propuesta por Riba (2002, p.178) en su texto Diseño Concurrente. A través de la casa 
de la calidad han sido determinados los requerimientos técnicos que satisfacen las necesidades del cliente, cada uno de los cuales, ha sido ponderado; aquellos de mayor valoración son los siguientes:

- Uso de visión artificial.- La máquina debe integrar el uso de visión artificial como herramienta para el cumplimiento de su función principal; esta es una demanda básica.

- Clasificación de objetos por forma y color.- Se trata de una característica básica ya que el cumplimiento del mismo es uno de los principales objetivos del proyecto y el no cumplirlo implicaría la insatisfacción total del cliente.

- Compacta.- Constituye un parámetro importante ya que el lugar destinado a la máquina es de espacio reducido, por lo cual es indispensable tomar en cuenta este parámetro dentro del diseño conceptual de la máquina.

- Proceso rápido.- Se trata de una demanda unidireccional, que con su mejora aumenta proporcionalmente la satisfacción del usuario.

\subsubsection{Especificaciones técnicas}

A partir del desarrollo de la casa de la calidad se obtienen las especificaciones técnicas que se muestran en la Tabla 1, las cuales constituyen el punto de partida para el diseño y desarrollo de la máquina.

Tabla 1. Especificaciones técnicas de la máquina clasificadora de objetos

\begin{tabular}{|c|c|c|c|}
\hline & \multirow{2}{*}{\multicolumn{2}{|c|}{$\begin{array}{l}\text { Producto: Máquina Clasificadora de } \\
\text { Objetos }\end{array}$}} & Fecha Revisión: \\
\hline & & & 12/10/2015 \\
\hline \multicolumn{4}{|c|}{ Especificaciones } \\
\hline Concepto & Propone & R/D & Descripción \\
\hline \multirow[t]{3}{*}{ Función } & $D+C$ & \multirow[t]{3}{*}{$D+R$} & Transporte de objetos \\
\hline & D & & Clasificación de objetos \\
\hline & $\mathrm{D}$ & & Almacenamiento de objetos \\
\hline \multirow[t]{2}{*}{ Movimientos } & & \multirow[t]{2}{*}{$D+R$} & Movimientos de traslación de objetos \\
\hline & C & & Movimientos de rotación de objetos \\
\hline Dimensión & C & D & Capacidad de 9 objetos diferentes \\
\hline \multirow[t]{3}{*}{ Mantenimiento } & $D+F$ & $\mathrm{D}$ & Documentación de montaje/desmontaje \\
\hline & $\mathrm{F}$ & D & Facilidad de transporte de los componentes. \\
\hline & $D+F$ & $\mathrm{R}$ & $\begin{array}{l}\text { Componentes asequibles en el mercado } \\
\text { nacional }\end{array}$ \\
\hline Energía & D & $\mathrm{D}$ & Energía eléctrica \\
\hline \multirow[t]{2}{*}{ Señales y control } & D & $D+R$ & Tablero de control \\
\hline & $D+C$ & $D+R$ & Sensores y actuadores \\
\hline \multicolumn{4}{|c|}{$\begin{array}{l}\text { Propone: } \\
\text { M=Marketing; C=Cliente; D=Diseño; P= Producción; F=Fabricación; R/D: R=Requerimiento; D=Deseo; MR=Modificación de } \\
\text { requerimiento; NR: Nuevo Requerimiento }\end{array}$} \\
\hline
\end{tabular}




\subsubsection{Definición de módulos}

Para establecer la división más apropiada para el producto en cuestión, se analiza cuidadosamente el diagrama funcional, tomando en cuenta las interfaces de los flujos de energía, material y las señales, en el caso de la máquina clasificadora de objetos se han determinado tres conjuntos principales de funciones o módulos que se muestran en la Figura 1.

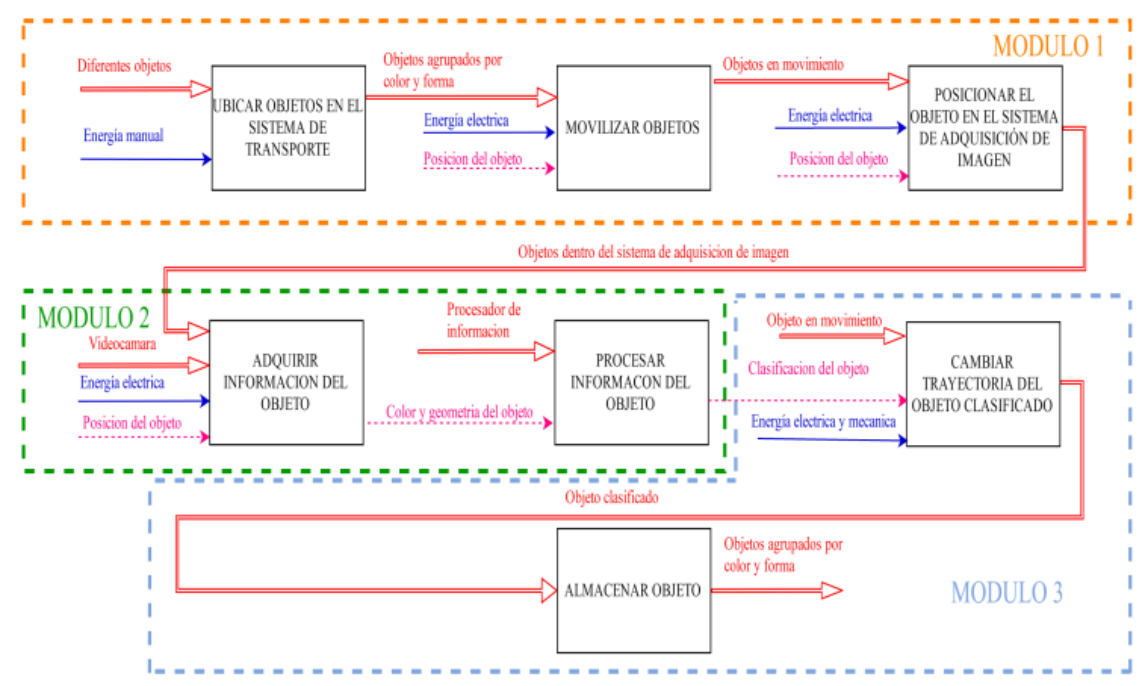

Figura 1. Diagrama funcional para la máquina clasificadora de objetos

Los módulos obtenidos y las soluciones a las funciones que lo componen mediante el uso de los criterios ponderados (Riba, 2002) son:

- Distribución de objetos: los objetos se posicionan manualmente en la banda transportadora perteneciente al sistema de transporte mostrado en la Figura 2.

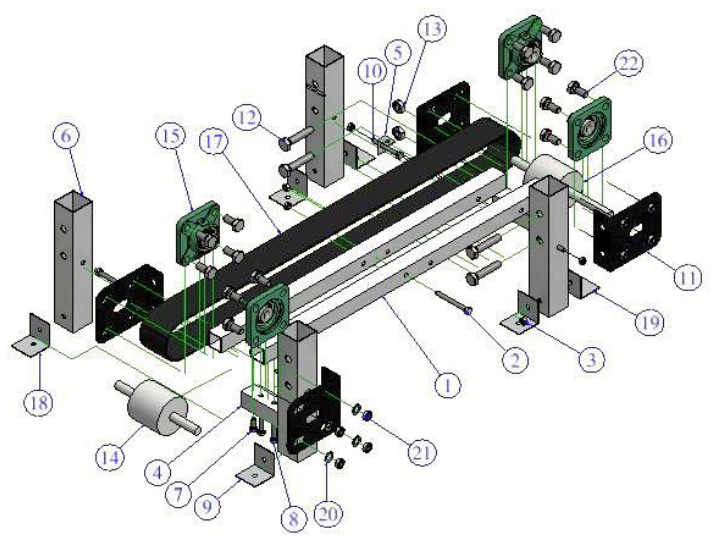

Figura 2. Sistema de transporte

- Procesamiento de información: los objetos se posicionan en el sistema de adquisición de imágenes mostrado en la Figura 3, a través de la banda transportadora; la imagen de la escena se lo realiza mediante una webcam y la información recopilada es procesada en el software LabVIEW. 


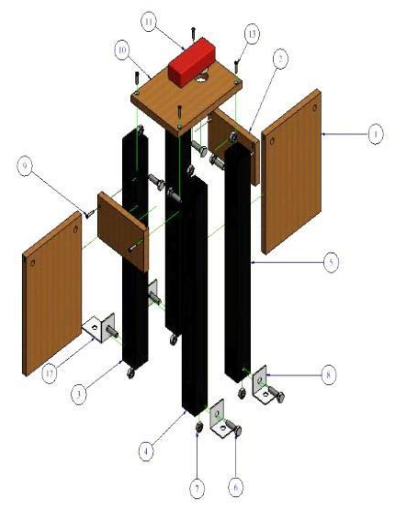

Figura 3. Sistema de adquisición de imágenes

- Ordenamiento de los objetos: los objetos son desviados y almacenados a través del uso de derivadores electrónicos y un plato contenedor giratorio como se muestra en la Figura 4.

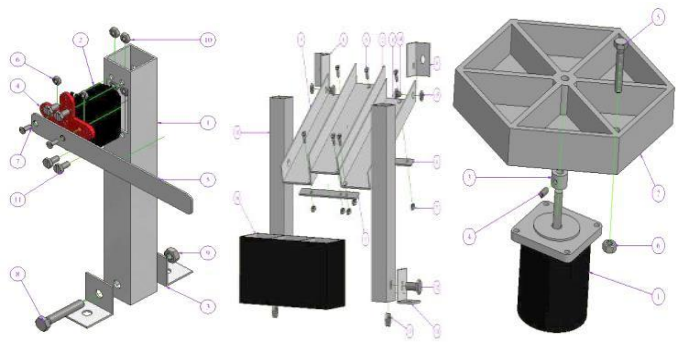

Figura 4. Sistema de almacenamiento de objetos

A partir del diseño conceptual se determinan las alternativas de solución para cada función o módulo cuya integración permite obtener el diseño conceptual de la máquina en su totalidad, tal como se muestra en la Figura 5.

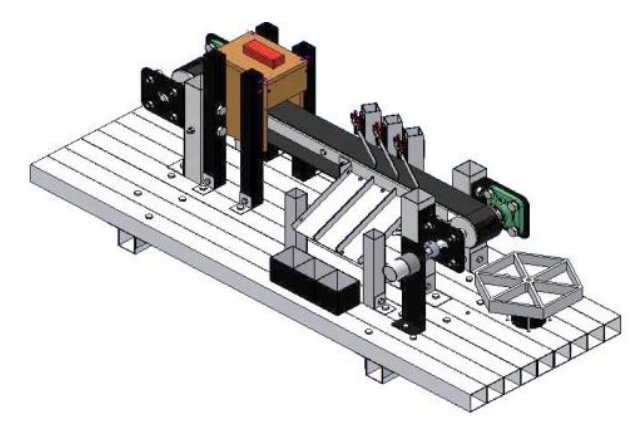

Figura 5. Esquema final de la máquina

\subsection{Visión artificial}

Una gran cantidad de actividades industriales se han beneficiado de las soluciones innovadores que ofrece la tecnología de visión artificial en los procesos de fabricación. La tecnología de visión artificial mejora la productividad y la gestión de calidad y proporciona una ventaja competitiva a las industrias que emplean esta tecnología (Schalkoff, 1999). 


\subsubsection{Adquisición y extracción de imagen}

El modelo de webcam empleado en el presente proyecto permitió obtener, a través de su controlador, diferentes resoluciones de imagen en valores intermedios desde los 120×160 [px] hasta $640 \times 480$ [px]. La adquisición de la imagen es realizada en tiempo real, a través del bloque Vision Acquisition, en donde las siguientes variables adquieren propiedades fijas establecidas por el programador:

- Controlador de la webcam

- Número de puerto de comunicación USB

- Resolución de la imagen

- Modo de adquisición de imagen (continuo)

\subsubsection{Segmentación y extracción de características}

La extracción del color del objeto en la escena se realizó a través del bloque IMAQ ColorLearn VI aplicada a una ROI (Region Of Interest) establecida por el usuario. Este bloque devuelve los valores de color detectados en la ROI en el espacio HSV, según la cantidad de matices de lo decidido por el usuario. Esta elección debe ser tal que permita abarcar los colores saturados de rojo, verde y azul en el disco $\mathrm{H}$ (hue); esto se consigue asignando el valor de low a la sensibilidad de color.

\subsubsection{Configuración del proceso de detección de coincidencias}

Se establecieron los parámetros para la configuración de la extracción de curvas de la imagen de inspección, los parámetros representativos corresponden al valor del umbral para la detección de contornos en la escena (edge threshold), la longitud mínima de una curva para ser incluida en el análisis (mínimum lenght), los rangos de rotación y escala de las curvas con respecto a la plantilla para la búsqueda de coincidencias y el mínimo valor para validar una curva como coincidencia (mínimum score). Estas asignaciones se muestran en la Tabla 2.

Tabla 2. Asignación de características de detección de forma

\begin{tabular}{|c|c|c|c|}
\hline Detección de forma & Triángulos & \multicolumn{2}{|c|}{\begin{tabular}{c} 
Riguras \\
\hline Modo de extraccióngulos
\end{tabular}} \\
\hline Umbral de borde & Normal & Región uniforme \\
\hline Tamaño de filtro de borde & 30 & Normal \\
\hline Longitud mínima & Normal & 63 \\
\hline Tamaño de la fila de búsqueda & 80 & 52 \\
\hline Tamaño de la columna de búsqueda & 50 & 64 \\
\hline Máxima abertura entre puntos finales & 50 & 30 \\
\hline
\end{tabular}




\subsubsection{Interpretación}

El procesamiento de la imagen culmina con la interpretación de las características obtenidas de la escena. Para el presente proyecto las características son:

- Triángulos: rojo, verde y azul.

- Cuadrados: rojo, verde y azul.

- Círculos: rojo, verde y azul.

\subsection{Algoritmos de detección de forma y color}

Los algoritmos con los cuales se trabaja en el reconocimiento (National Instruments, 2013) y la detección de objetos se muestran a continuación.

\subsubsection{Técnica geométrica de emparejamiento}

Esta técnica se encarga de buscar en la imagen a analizar información similar a la presente en una plantilla base. Después de la simplificación de la información, se extraen características las cuales describen a la imagen (Figura 6). Este procedimiento consta de dos fases: aprendizaje y emparejamiento de imágenes. En el proceso de aprendizaje, el algoritmo guarda las características presentes en la imagen a ser analizada, guarda los datos en espacios de memoria para que en lo posterior, se realice una búsqueda organizada.

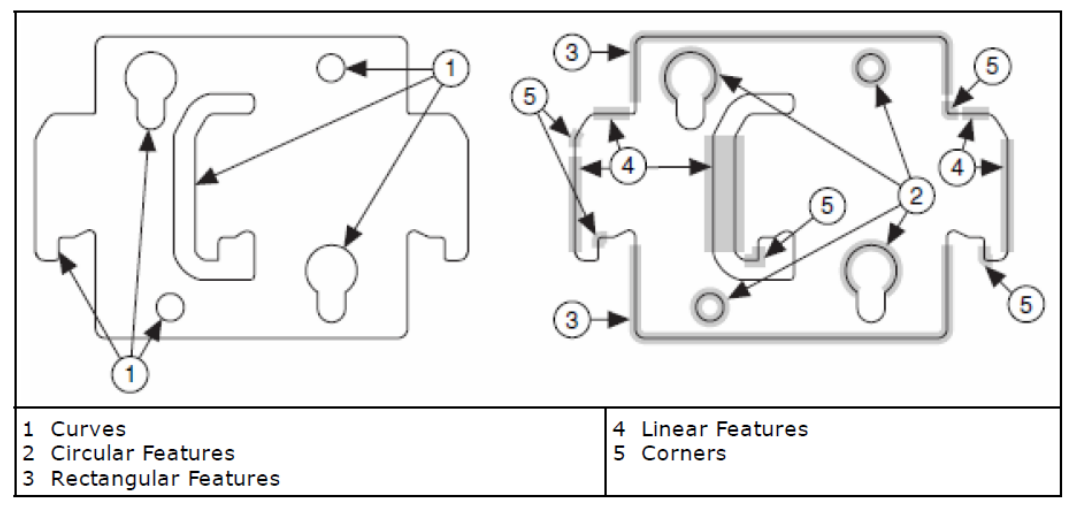

Figura 6. Bordes característicos para extracción en una imagen (National Instruments, 2013).

\subsubsection{Extracción de curvas}

En el proceso de extracción de curvas, es necesario encontrar un punto semilla el cual será el encargado de dar origen al proceso de extracción. El borde de forma curvilínea se procesa computacionalmente de acuerdo con la intensidad del mismo y la de sus respectivos pixeles vecinos. Si $P_{(i, j)}$ es el pixel de análisis, el borde del contraste se define por (Ecuación 1):

$$
\text { Contraste del borde }=\sqrt{\left(P_{(i-1, j)}-P_{(i+1, j)}\right)^{2}+\left(P_{(i, j-1)}-P_{(i, j+1)}\right)^{2}}
$$


Donde:

$P_{(i-1, j)}, P_{(i+1, j)}, P_{(i, j-1)}$ y $P_{(i, j+1)}:$ píxeles vecinos a $P_{(i, j)}$

\subsubsection{Técnica de emparejamiento geométrico basado en bordes}

En el proceso de extracción de borde se realiza el procesamiento del gradiente de la imagen en cada punto del contorno de análisis. Las herramientas con las cuales se realiza el procesamiento a través de la transformada de Hough y la generación de la tabla R (Figura 7).

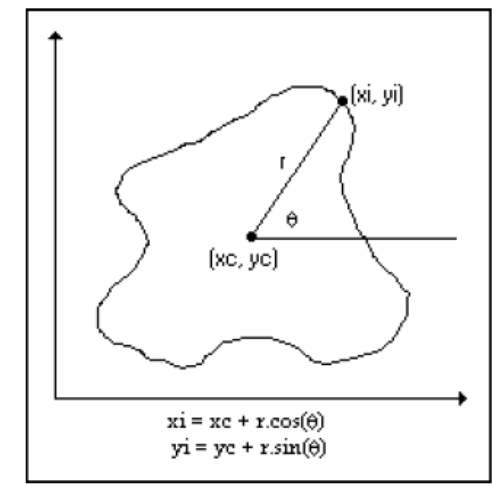

Figura 7. Mapeo de pixeles en una imagen (National Instruments, 2013).

El algoritmo se realiza a través de las siguientes etapas:

1. El algoritmo selecciona el centro de la imagen como puntos de referencias (xc, yc).

2. Para cada punto ( $x i, y i)$ perteneciente a las curvas de la imagen, se calcula la distancia $y$ orientación del punto (ri, $\theta \mathrm{i})$ de estudio con respecto a (xc, yc).

3. El algoritmo guarda los datos de (ri, $\theta i)$ en la tabla $R$ con todos los puntos del contorno de la imagen.

Tabla 3. Gradientes tomados en el análisis de una imagen

\begin{tabular}{|c|c|}
\hline Gradiente $(\varnothing)$ & Valores \\
\hline$\varnothing 1$ & $(r 1, \theta 1),(r 4, \theta 4)$ \\
\hline$\varnothing 2$ & $(r 2, \theta 2),(r 10, \theta 10)$ \\
\hline$\varnothing n$ & $(r n, \theta n),(r i, \theta i)$ \\
\hline
\end{tabular}

\subsubsection{Método del gradiente}

El presente método utiliza pixeles a los cuales se les ha sometido a un proceso de filtrado. Los bordes de la imagen son procesados a partir de una conversión de la imagen original a escala de grises en la cual la intensidad de la imagen se obtiene a través del gradiente del pixel en análisis (Figura 8). 


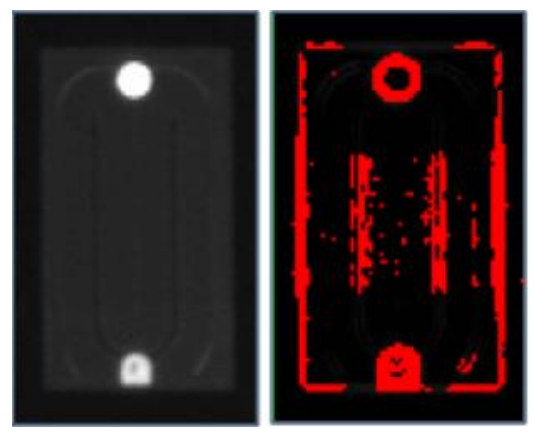

Figura 8. Procesamiento de una imagen a través del método del gradiente (National Instruments, 2013)

\subsection{Comunicación LabVIEW 2015 - PLC S7-1200}

El software $\mathrm{NI}$ LabVIEW puede comunicarse con cualquier controlador lógico programable (PLC) de diferentes formas. El servidor OPC define el estándar para comunicar datos en tiempo real entre los dispositivos de control y las interfaces hombre-máquina (HMI) (Valencia, 1999).

La conexión del computador con OPC a un PLC Siemens S7-1200 a través de Ethernet (Valencia, 1999) se establece mediante el uso del módulo DSC (Datalogging and Supervisory Control) del software LabVIEW siguiendo el esquema mostrado en la Figura 9.

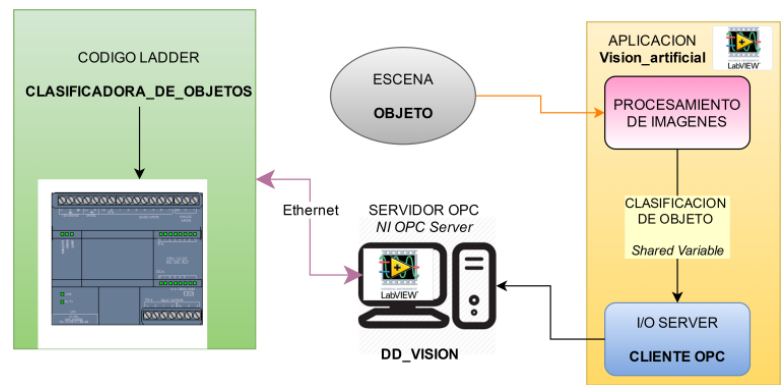

Figura 9. Comunicación entre la aplicación de visión artificial y el PLC S7-1200 mediante OPC

\subsection{Diseño y selección de componentes electrónicos}

La interfaz para la comunicación de instrucciones entre el PLC y los controladores de los actuadores se realiza a través de una tarjeta electrónica. El diseño de la tarjeta electrónica integra las siguientes características:

- Suministro de energía a los componentes eléctricos y electrónicos

- Control del motor-reductor a través del PLC

- Comunicación de las señales de los sensores hacia el PLC

Los requerimientos de energía eléctrica que se muestran en la Tabla 4 son suplidos mediante la implementación de una fuente ATX de 750 [W]. 
Tabla 4. Requerimientos energéticos de la máquina

\begin{tabular}{|c|c|}
\hline COMPONENTE & VOLTAJE [V] \\
\hline MOTOR-REDUCTOR DC & 3.3 \\
\hline ILUMINACIÓN LED & 12 \\
\hline CONTROLADOR MOTOR PASO A PASO & 5 \\
\hline ALIMENTACIÓN SERVOMOTORES & 5 \\
\hline ALIMENTACIÓN PLACA ARDUINO MEGA 2560 & 5 \\
\hline
\end{tabular}

Se emplea un tablero central de mando a través de una interfaz que comunica al PLC y a la tarjeta Arduino MEGA 2560 cuenta con una conexión mediante puerto DB25 para la comunicación de señales de entradas y salidas hacia el PLC (Artero, 2013).

La interfaz para la obtención de su circuito impreso atiende el esquema mostrado en la Figura 10.

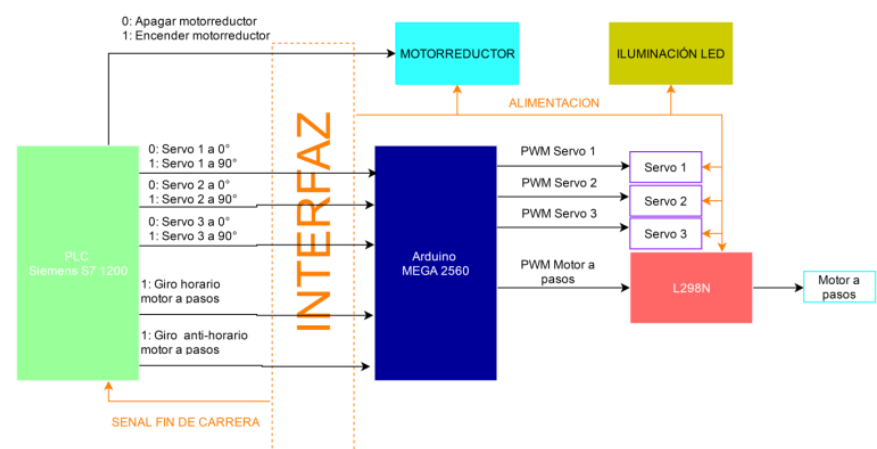

Figura 10. Entorno de la interfaz gráfica

\subsection{Diseño y selección de elementos mecánicos}

Los elementos mecánicos que constituyen estructuras de soporte fueron diseñados a partir de perfiles de aluminio 6061, para los cuales los esfuerzos generados por los elementos motrices no superan por un gran margen su límite de falla (Shigley. 2011).

\subsubsection{Selección de la banda transportadora}

Para la selección de la banda transportadora, se debe determinar si el material de esta es capaz de resitir los esfuerzos generados por la fuerza de tracción. Para este fin se procede con la metodología propuesta por Forbo (2009).

$$
\begin{gathered}
\frac{F_{1}}{b_{o}} \leq C_{2}(2) \\
C_{2}=\varepsilon_{\max } \times k_{1 \%}(3)
\end{gathered}
$$

Donde:

$F_{1}$ : fuerza de tracción máxima de la banda, $2,13 \mathrm{~N}$

$b_{o}$ : ancho de la banda, $50 \mathrm{~mm}$

$\varepsilon_{\text {max }}$ : elongación máxima de la banda, 2 (Forbo, 2009, p.4) 
$k_{1 \%}$ : fuerza de tracción relajada de la banda con una elongación 1\% por unidad de anchura, 30 $\mathrm{N} / \mathrm{mm}$

La especificación de la banda que cumple con las solicitaciones mecánicas planteadas es Febor F30 CFy as dimensiones finales de la banda se muestran en la Figura 11.

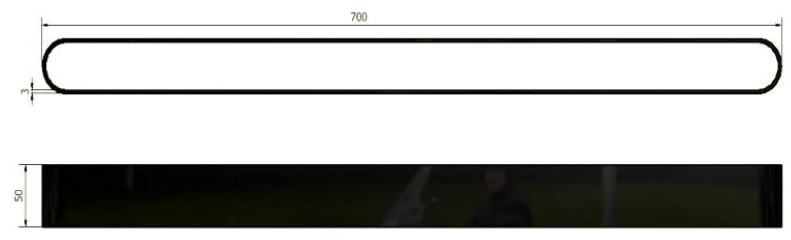

Figura 11. Dimensiones de la banda

\subsubsection{Dimensionamiento del tambor motriz}

El dimensionamiento del tambor motriz se lo realiza en base a la metodología propuesta por Forbo (2009).

Para el diámetro mínimo del tambor motriz se utiliza la Ecuación 4 y se sigue:

$$
d_{A}=\frac{F_{U} \times C_{3} \times 180^{\circ}}{b_{o} \times \beta}
$$

Donde:

$d_{A}: \quad$ diámetro mínimo del tambor motriz, en mm

$F_{U}: \quad$ fuerza tangencial, $1,42 \mathrm{~N}$

$C_{3}: \quad$ factor de cálculo válido para rodillo motriz, 25 (Forbo, 2009, p.5)

$b_{o}$ : ancho de banda, $50 \mathrm{~mm}$

$\beta$ : $\quad$ ángulo de contacto, $180^{\circ}$

El diámetro mínimo del tambor motriz es de 0,71 mm; sin embargo, se asigna un valor de $45 \mathrm{~mm}$ debido a aspectos constructivos.

Los tambores y los rodillos deben ser lo suficientemente anchos para asegurar que la totalidad de la superficie de la banda se halle en contacto con estos; para este fin Habasit (2010) recomienda los valores mostrados en la Tabla 5.

Tabla 5. Recomendaciones para las dimensiones del tambor

\begin{tabular}{|c|c|}
\hline $\begin{array}{c}\text { Ancho de la banda (bo) } \\
\text { bo } \leq \mathbf{1 0 0}[\mathrm{mm}]\end{array}$ & Ancho del tambor (b) \\
\hline bo $>\mathbf{1 0 0}[\mathrm{mm}]$ & $\mathrm{b}=(1,08 \times \mathrm{bo}+20[\mathrm{~mm}]$ \\
\hline
\end{tabular}

Por consiguiente el valor del ancho del tambor motriz es de $70 \mathrm{~mm}$. 


\subsubsection{Selección de chumaceras}

La selección de las chumaceras se realiza de tal manera que estas resistan la capacidad de carga estática generada por las cargas y fuerzas resultantes en los ejes de los tambores.

La capacidad de carga estática se calcula a partir de la Ecuación 5 (SKF, 2016):

$$
C_{0}=S_{0} \times P_{0}(5)
$$

Donde:

$C_{0}$ : capacidad de carga estática, en $\mathrm{N}$

$P_{0}$ : carga estática equivalente, $1,42 \mathrm{~N}$

$S_{0}$ : factor de seguridad estático, 1.3

Se selecciona una chumacera UCF201-8 de la compañía CMB con rodamiento de bolas UC201-8 con capacidad de carga estática de $6650 \mathrm{~N}$, la misma que garantiza el valor de carga estática necesario y el diámetro del eje del tambor asequible en el mercado local.

Posterior al análisis y dimensionamiento de elementos del módulo didáctico, el resultado final se aprecia en la Figura 12:

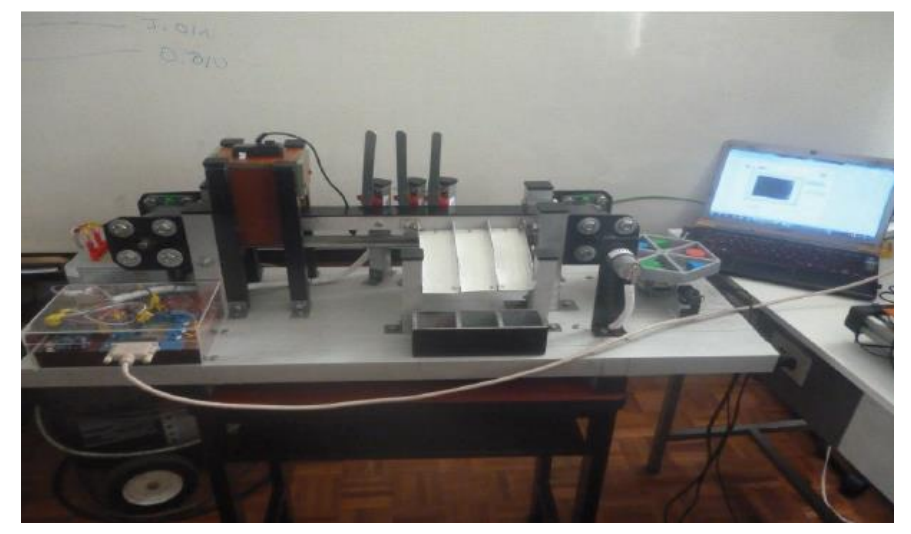

Figura 12. Módulo final ensamblado

\section{Resultados y discusión}

El funcionamiento de la máquina se aprecia a través del diagrama de flujo de la Figura 13.

El diseño de la máquina atiende las necesidades del usuario a través de compromisos técnicos establecidos; aquellos que requieren un mayor interés son: la capacidad de depósito y los objetos almacenados por minuto, la capacidad de depósito y el volumen, el nivel de automatización y el tipo de tecnología, ya que estos suponen un $52,5 \%$ del total de incidencias significativas que mejoran el producto. Una vez establecido el diseño, se procede a la materialización mediante la construcción del prototipo de la máquina. Los ensayos realizados para verificar el funcionamiento de la máquina se presentan a continuación. 


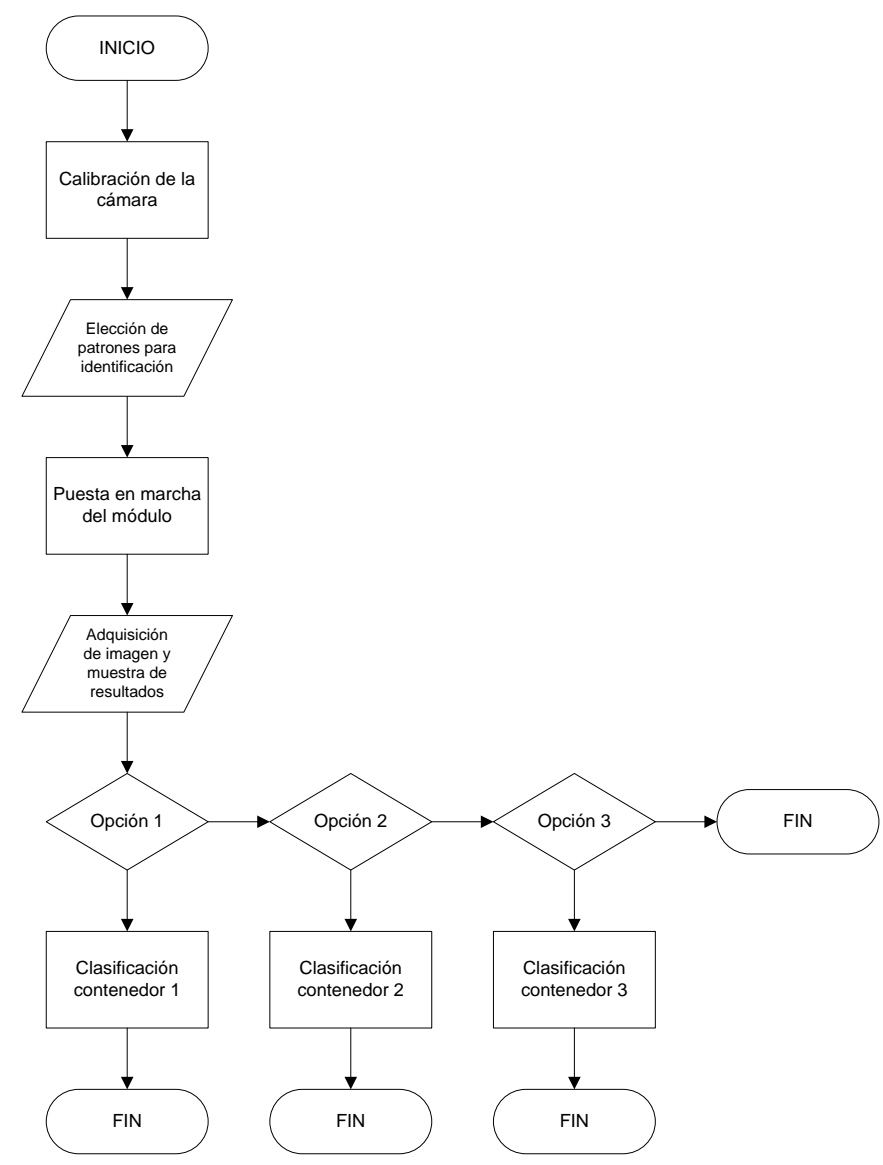

Figura 13. Diagrama de flujo del funcionamiento del módulo

\subsection{ENSAYO 1}

\section{OBJETIVO}

Verificar con base en las especificaciones técnicas propuestas para el diseño, los siguientes aspectos de la máquina: documentación de ensamblaje, componentes asequibles, facilidad de transporte de componentes.

\subsection{ENSAYO II}

\section{OBJETIVO}

Verificar con base en las especificaciones técnicas propuestas para el diseño, los siguientes aspectos de la máquina: movimientos de traslación de objetos, movimientos rotativos de objetos.

\subsection{ENSAYO III}

\section{OBJETIVO}

Verificar con base en las especificaciones técnicas propuestas para el diseño los siguientes aspectos de la máquina: capacidad de almacenar 9 objetos diferentes. 


\subsection{ENSAYO IV}

Verificar con base en las especificaciones técnicas propuestas para el diseño, los siguientes aspectos de la máquina: transporte, clasificación y almacenamiento de objetos, sensores, actuadores y tablero de control.

Tras cumplirse exitosamente los 4 ensayos realizados en el módulo didáctico, se concluye que el sistema de clasificación posee la funcionalidad necesaria para la cual fue creado.

\section{Conclusiones y recomendaciones}

Se desarrolló la máquina didáctica para agrupar 9 objetos determinados de diferente geometría y color en espacios individuales mediante el uso de visión artificial utilizando los criterios de diseño proporcionados por el usuario, tomando en cuenta criterios de enseñanza y aprendizaje. Se concatenaron elementos electrónicos, mecánicos y sistemas de información para la elaboración del módulo mecatrónico.

Se implementó la técnica de detección de bordes para la clasificación de objetos por la forma. En cambio, para la clasificación de objetos por el color, la técnica debe ser cambiada a un método de detección por patrones.

Se realizó la comunicación de datos en alta velocidad entre el software de procesamiento de imágenes y el PLC fue establecida de manera adecuada a través de una conexión Ethernet gestionada mediante el servidor OPC proporcionado en el software LabVIEW. Pese a la inclusión de varios elementos como: PLC, Arduino, cámara de adquisición de datos, el retardo generado fue de baja latencia y el proyecto tiene la funcionalidad requerida por el usuario.

Para futuros proyectos a fines a la visión artificial se recomienda experimentar con cámaras USB con mejores características y un adecuado sistema de iluminación.

También, se recomienda desarrollar una nueva metodología para establecer la comunicación entre un software para el procesamiento de imágenes y un PLC que sea validado mediante el uso de la máquina clasificadora desarrollada en el presente trabajo.

\section{Bibliografía}

Alcalde Cajamarca, C. F., \& Bone Fonte, E. G. (2013). Diseño de una máquina clasificadora de tomates de $700[\mathrm{Kg} / \mathrm{h}]$ de capacidad. Quito; EPN.

Bolton, W. (2001). Mecatrónica: sistemas de control electrónico en la ingeniería mecánica y eléctrica. Marcombo.

Cintas, P. G., \& Llabres, J. T. M. (1995). Técnicas para la gestión de la calidad. Ediciones Díaz de Santos. 
Forbo. (2009). Siegling transilon: Bandas de transporte y procesamiento. Grupo Forbo Siegling.

Habasit. (2010). Habasit: Cintas transportadoras con estructura de tejido, guía de ingeniería. Habasit Solution in motion.

National Instruments. (2011). Support: Geometric Matching Technique. Recuperado de: http://www.ni.com/es-cr.html

National Instruments. (2013). Support: Pattern Matching Techniques. Recuperado de: http://www.ni.com/es-cr.html

NI Vision 2013 Concepts Help - National Instruments. (2017). Zone.ni.com. Recuperado 26 April 2017, a partir de http://zone.ni.com/reference/en-XX/help/372916P-01/

Riba i Romeva, C. (2002). Diseño concurrente. Barcelona: Edicions UPC.

Schalkoff, R. J. (1989). Digital image processing and computer vision (Vol. 286). New York: Wiley.

Shigley, J. E. (2011). Shigley's mechanical engineering design. Tata McGraw-Hill Education.

SKF. (2016). Productos: Capacidad de carga estática requerida. Recuperado de: http://www.skf.com/

SKF. (2016). Productos: Carga estática equivalente. Recuperado de: http://www.skf.com/

Stemmer Imaging. (2016). Learn: Technical tips. Recuperado de: http://www.stemmerimaging.co.uk/en/

Valencia Villacís, E. J. (1999). Interfaz de software entre un computador personal y un PLC, mediante un drive de comunicación y su aplicación en el control y monitoreo de un invernadero. Quito; EPN. 